\section{Corneal shape change during accommodation}

\begin{abstract}
Purpose To investigate whether accommodation induces any changes in central corneal curvature.

Methods Shape changes were measured on 14 subjects using a keratometer modified to enable a change in focus to occur without a change in vergence. All subjects were emmetropic and their ages ranged from approximately 20 to 28 years.

Results In 11 of the 14 subjects a difference in central corneal curvature, of around $0.4 \mathrm{D}$ in at least one principal meridian, was found when focus was changed between distant and near targets. In 9 subjects the curvature was greater for near focus in at least one meridian. In $\mathbf{5}$ subjects the change in one meridian was opposite in effect to what would be expected, i.e. the curvature was greater at distance than at near.

Conclusion The study suggests that accommodation may have some effect on corneal shape.
\end{abstract}

Key words Accommodation, Cornea, Keratometry, Shape change

Accommodation is the dynamic focusing process attributed to the eye lens. The change in lens shape, mediated by the action of the ciliary muscle, is indisputably the mechanism which allows the pre-presbyopic eye to meet visual demands over a range of distances. The cornea, which contributes by far the greater share of

A. Popiolek-Masajada

H. Kasprzak

Wroclaw University of

Technology

Institute of Physics

Wybrzeże Wyspiańskiego 27

50-370, Wrodlaw,

Poland

B.K. Pierścionek

Department of Biomedical

Sciences

University of Bradford

Bradford

West Yorkshire BD7 1DP, UK

e-mail:

b.pierscionek@bradford.ac.uk

Received: 8 January 2001 Accepted in revised form: 13 August 2001
B.K. PIERŚCIONEK,

A. POPIOLEK-MASAJADA, H. KASPRZAK measurable. It would be almost certainly masked by any pulling forces which may be exerted on the cornea by the horizontal recti during the convergence which accompanies accommodation. ${ }^{6}$ This paper reports findings from a study which sought to investigate whether it was possible to detect any change in corneal shape arising purely from

accommodation. The measurements were taken in the primary gaze position in order to avoid convergence.

\section{Materials and methods}

It is important to point out that the instrumental modifications and incorporation of the additional fixtures necessary for this experiment could not be made on any instrument which measures corneal topography because of the solid structure of the 'bowl'. A Javal keratometer was modified, as shown in Fig. 1, to allow a change in focus of the eye without any motion. This was checked by the observer, who ensured that measurements were taken only when there were no shifts in position of the keratometric mires. Two positions of fixation were chosen: one at $11 \mathrm{~cm}$ from the corneal vertex and one beyond $6 \mathrm{~m}$. In order to change focus without inducing any change in vergence, the two fixation points for the eye undergoing testing were arranged in such a way that they could be superimposed. This was achieved by placing the near target (a letter ' $\mathrm{O}$ ') at the edge of a microscope slide which was placed in front of the eye and tilted so that it did not obscure the observer's view of the cornea. The size of this letter corresponded to N12 capital letter size. The distance target (a letter ' $X^{\prime}$ ) was placed $3 \mathrm{~m}$ behind the subject's head and reflected by a mirror at the side onto the slide so that its reflected image superimposed the near target: the reflected image of the distant letter ' $X$ ' fitted into the near letter ' $\mathrm{O}$ '. The microscope slide was sufficiently reflective to be used in place of a second mirror, as it was lighter, less obstrusive and not distracting to the subject. In this way the subjects were able to change focus from one target to the other without any change in eye position. The eye which was not tested was covered.

Subjects were given $5 \mathrm{~min}$ to practice the technique and then $2 \mathrm{~min}$ to relax with their eyes closed. All were sufficiently competent 


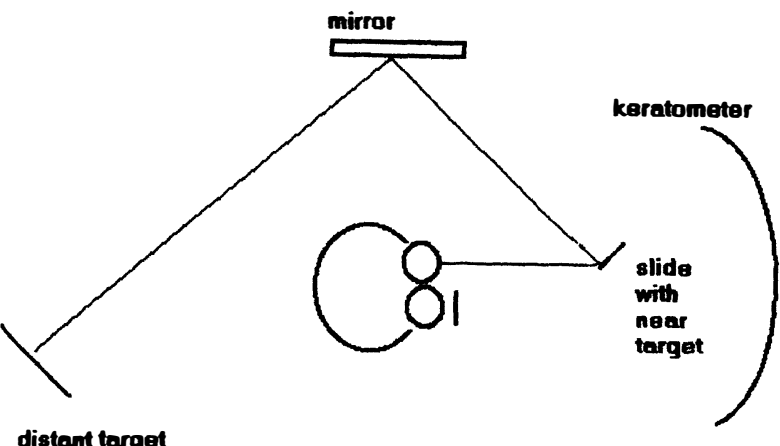

Fig. 1. Diagrammatic representation showing the arrangement used for taking keratometric readings for two separate focusing positions, without inducing convergence.

within the practice time to alter focus without any eye motion. All cycles started with focus on the distant target and after several seconds the experimenter asked the subject to change focus to the near object with a singleword instruction. When the focus of the instrument was adjusted to the accommodative change, the subject was once again instructed with a single word to alter focus to the distant target. Each subject was asked to change focus from distance to near 4 times, i.e. results for 4 complete cycles of change were collected from every subject. Power was always measured along the two principal meridians of $90^{\circ}$ and $180^{\circ}$. The differences in power between corresponding near and distant focusing pairs were averaged and standard deviations calculated.

Fourteen emmetropic subjects ranging in ages from 237 to 334 months (19.75 years to 27.8 years) were used in this study. All were students at Wroclaw University of Technology. Three men and 11 women took part in the study.

\section{Results}

From the differences in readings between near and distance focusing points (Table 1), it can be seen that for all subjects most of the differences between the focusing points vary between 0.3 and 0.5 dioptres for both meridians. Four subjects showed no change at one of the meridians. It is important to note that in all the subjects there was some alteration at one or both meridians, i.e. for all subjects some change in corneal shape occurred with alteration of focus.

In 5 cases the difference between near and distance readings for one of the meridians was negative, indicating that the power in that meridian was greater for distant focus. As near focusing demands the greater power, this appears to be inconsistent with visual needs. Only 2 of these 5 cases (subjects 3 and 7) showed an overall increase in power with distance focusing. There was nothing unusual about either of these cases. In the absence of other evidence, one can speculate that there may have been a mistake in following instructions, so that the subjects focused at the near when they should have focused on the distant target and vice versa, or that there was a longer lag time in these 2 subjects in adjusting to the focal distances than the experimenter had allowed. The differences in power between distance and near focusing for the two cylinder powers are compared in the final column of Table 1. Six subjects showed no predominant change in either meridian. In subjects $3,5,9$ and 10 the greater change in corneal shape with change of focus occurred in the $180^{\circ}$ meridian, whereas in subjects $4,6,7$ and 14 the greater change in shape with focus alteration was in the $90^{\circ}$ meridian.

\section{Discussion}

The purpose of this study was to investigate whether accommodation of the lens had any measurable effect on the shape of the cornea. Anatomically, it is feasible that movement of the ciliary muscle could exert some slight effect on the lens, as some of the muscle fibres are in contact with the anterior sclera which in turn extends into the cornea. ${ }^{7}$ Subjects chosen for this study were young, educated adults with ample accommodation to be able to physically perform the task and sufficient intelligence to understand and correctly follow the instructions. The accommodative effort asked of the

Table 1. Differences between keratometry readings taken for near and distant targets at each of the two principal meridians

\begin{tabular}{|c|c|c|c|c|c|c|}
\hline \multirow[b]{2}{*}{ Subject no. } & \multicolumn{4}{|c|}{ Difference in power between near and distance targets (D) } & \multicolumn{2}{|c|}{$\begin{array}{l}\text { Power difference at } 90^{\circ}- \\
\text { power difference at } 180^{\circ}\end{array}$} \\
\hline & $180^{\circ}$ & SD & $90^{\circ}$ & SD & Difference & SD \\
\hline 1 & 0.42 & \pm 0.16 & 0.50 & \pm 0.06 & 0.08 & $\pm 0.22^{*}$ \\
\hline 2 & 0.37 & \pm 0.11 & 0.50 & \pm 0.00 & 0.13 & $\pm 0.11^{*}$ \\
\hline 3 & 0.00 & $\pm 0.00^{*}$ & -0.45 & \pm 0.14 & -0.45 & \pm 0.14 \\
\hline 4 & -0.28 & \pm 0.06 & 0.32 & \pm 0.12 & 0.60 & \pm 0.18 \\
\hline 5 & 0.50 & \pm 0.00 & 0.40 & \pm 0.00 & -0.10 & \pm 0.00 \\
\hline 6 & -0.46 & \pm 0.09 & 0.32 & \pm 0.16 & 0.78 & \pm 0.25 \\
\hline 7 & -0.50 & \pm 0.21 & 0.05 & $\pm 0.06^{*}$ & 0.55 & \pm 0.27 \\
\hline 8 & 0.3 & \pm 0.11 & 0.44 & \pm 0.12 & 0.09 & $\pm 0.23^{*}$ \\
\hline 9 & 0.57 & \pm 0.19 & -0.25 & \pm 0.00 & -0.82 & \pm 0.19 \\
\hline 10 & 0.40 & \pm 0.05 & -0.11 & $\pm 0.22^{*}$ & -0.54 & \pm 0.27 \\
\hline 11 & 0.25 & \pm 0.0 & 0.25 & \pm 0.0 & 0.00 & $\pm 0.00^{*}$ \\
\hline 12 & 0.50 & \pm 0.16 & 0.37 & \pm 0.12 & -0.13 & $\pm 0.28^{*}$ \\
\hline 13 & 0.58 & \pm 0.12 & 0.44 & \pm 0.11 & -0.14 & $\pm 0.23^{*}$ \\
\hline 14 & 0.00 & $\pm 0.00^{*}$ & 0.58 & \pm 0.12 & 0.58 & \pm 0.12 \\
\hline
\end{tabular}

*Indicates no change. 
subjects was large (approximately 9 D) and therefore at the limit of their capacity; the resulting change measured was small (around 0.5 D). However, it should be remembered that the measurement was made over the central cornea, where any residual shape change translated to the sclera from the pull of the ciliary muscle would be least evident. The mere fact that some change was noted in the centre, the point furthest from the force which instigated the shape change, suggests that a greater change in shape would have been found in the periphery. In terms of effect on vision, this peripheral change is of course of little, if any, consequence. What is of significance is that the cornea possesses sufficient malleability to deform under the action of the ciliary muscle. Previous studies have reported changes in corneal topography following extraocular muscle ${ }^{8,9}$ and scleral buckling ${ }^{10}$ surgery. The latter study showed that the type of shape change to the cornea depended on the surgical method used. Kwitko et al. ${ }^{8}$ showed, from experiments on rabbit eyes, that recession of an extraocular muscle causes flattening of the cornea in the quadrant of that muscle. A study on strabismus surgery conducted on patients with Graves' disease ${ }^{9}$ lent support to this. The suggestion was made that corneal topography may be altered either as a result of extraocular muscle tension or because of intraocular pressure.

The measurements were made for the principal meridians of $90^{\circ}$ and $180^{\circ}$ as no subjects showed any deviation from these directions in the toricity of their corneas. Accommodation, though, is not restricted to a shape change along these two meridians. This means that any shape change in the cornea, resulting from accommodation, should be evident in meridians other than the two measured. In this study, measurements were kept to a minimum as the task was onerous and accuracy in two meridians was preferred to erroneous measurements in a greater number of directions.

Although it is commonly assumed that the force of the ciliary muscle's pull on the lens has an even radial distribution, the effect of accommodation on the cornea would not be expected to follow this pattern. The cornea has a densely packed structure which would tend to resist radial stretching. Any mechanical force acting to cause steepening in one meridian would produce flattening in the perpendicular meridian. Moreover, there have been suggestions of a disproportionate shape alteration of the lens during accommodation, resulting in an astigmatic rather than a spherical change, and this has been referred to as astigmatic or meridional accommodation. ${ }^{11-13}$ In all but one of the subjects, as focus was altered the change in refractive power, and therefore the change in curvature, was unequal in the two meridians measured. The effort of accommodation therefore did not affect the two meridians to the same extent. This may be a manifestation of an uneven distribution of ciliary muscle action. Alternatively, it may be related to the asymmetry of the cornea, the vertical diameter of which is smaller than the horizontal. If the asymmetry of the cornea was the primary reason for the unequal changes in the two meridians, it could be expected that the differences would be consistent in all or most subjects. The horizontal diameter being the greater, any effect of accommodation on the cornea should therefore have been smaller in the $180^{\circ}$ meridian than in the $90^{\circ}$ meridian. The results do not support this. Four subjects showed a greater effect of accommodation on the $180^{\circ}$ corneal meridian and the same number showed a greater effect of accommodation on the $90^{\circ}$ meridian (Table 1). In the majority of subjects there was no difference in the effect of accommodation on either meridian. Hence, the corneal diameter appears not to have a predominant effect on the extent of mechanical deformation when this is induced by the action of the ciliary muscle.

If, indeed, the cornea has sufficient malleability to show that, with accommodation, an alteration of shape is measurable, even at the centre of the cornea, one is tempted to suggest that further studies looking at the effect on corneal shape of certain eye exercises, based on carefully and appropriately chosen combinations of external and internal ocular muscle movements, are worthy of investigation. The effects of the extraocular muscles on corneal shape can be predicted because each of these muscles has a defined direction of action. The effect of the ciliary muscle on corneal shape will require further study as the ciliary muscle exerts a radial force which may or may not be equally distributed in all directions. Subsequent work will need to consider how the corneal topography is altered by accommodation in different gaze positions, in order to assess the combined effects of the ciliary muscle action with the actions of each of the extraocular muscles.

It has been reported that the corneal shape shows diurnal variations. ${ }^{14}$ These tend to be a steepening, from morning to the evening, at a position $0.5 \mathrm{~mm}$ from the corneal apex (of around $0.36 \mathrm{D}$ ) and in the inferotemporal quadrant (of around $0.28 \mathrm{D}$ ). The magnitude of these changes is of the same order of magnitude as the change found in this study. Eyes which had undergone radial keratotomy also showed corneal steepening from morning to evening but over a wide area of the cornea. ${ }^{14}$ No effect of intraocular pressure, corneal thickness, gender, number of incisions, clearzone size of post-operative period on the diurnal variation were found. The effects of accommodation were not considered.

This study was conducted on subjects within the same age group. This choice of subjects was deliberate; intelligent, mature individuals with sufficiently high accommodative capacity were required. In addition, all subjects were emmetropic. The results cannot therefore be considered, at this stage, to be representative of all age groups and are likely to differ in cases where refractive error is high. For example, high myopes with low scleral rigidity may show greater effects of accommodation on the corneal shape.

The findings from this study point to a need to investigate further the relationship of lenticular dynamics on the cornea. Even the small degree of 
'corneal accommodation', as demonstrated in this study, may provide a useful physiological function for pseudophakic patients. It was only possible, thus far, to use a keratometer which provides information restricted to the central cornea. Nevertheless, what has been shown is that this central area, which is least likely to be affected by the action of the ciliary muscle, does show measurable changes. This indicates that a more extended study of corneal topography change with change in accommodation is worth investigating. Current instruments do not permit the kind of modifications needed for allowing change of focus without any change in vergence. A design with a more flexible structure is needed.

This work was made possible because of a grant from the British-Polish Joint Research Collaboration Programme supported jointly by the British Council and KBN (Komitet Badan Naukowych).

\section{References}

1. Lyle WM. Changes in corneàl astigmatism with age. Am J Optom Arch Am Acad Optom 1971;48:467-78.

2. Morgan MW. Changes in refraction over a period of twenty years in a non-visually selected sample. Am J Optom Arch Am Acad Optom 1958;35:281-300.

3. Phillips RA. Changes in corneal astigmatism. Am J Optom Arch Am Acad Optom 1952;29:379-80.
4. Hayashi K, Hayashi H, Hayashi F. Topographic analysis of the changes in corneal shape due to aging. Cornea 1995;14:527-32.

5. Reading VM. Corneal curvature. Contact Lens 1972;3:23-5.

6. Marin Amat M. Les variations physiologiques de la courbure de la cornée pendant de vie: leur importance et transcendance dans la réfraction oculaire. Bull Soc Belge Ophtalmol 1956;113:251-93.

7. Warwick R. Eugene Wolff's anatomy of the eye and orbit, 7th ed. London: HK Lewis, 1976.

8. Kwitko S, Sawusch MR, McDonnell PJ, Gritz DC, Moreira H, Evensen D. Effect of extraocular muscle surgery on corneal topography. Arch Ophthalmol 1991;109:873-8.

9. Kwitko S, Feldon S, McDonnell PJ. Corneal topographic changes following strabismus surgery in Graves' disease. Cornea 1992;11:36-40.

10. Hayashi H, Hayashi K, Nakao F, Hayashi F. Corneal shape changes after scleral buckling surgery. Ophthalmology 1997;104:831-7.

11. Fletcher RJ. Astigmatic accommodation. Br J Physiol Opt 1951;8:73-94.

12. Hallden $U$, Henriesson M. Astigmatism of the lens by asymmetric contraction of the ciliary muscle. Acta Ophthalmol 1974;52:242-5.

13. Brzezinski MA. Astigmatic accommodation (sectional accommodation): a form of dynamic astigmatism [review]. Aust J Optom 1982;65:5-11.

14. Kwitko S, Gritz DC, Garbus JJ, Gauderman WJ, McDonnell PJ. Diurnal variation of corneal topography after radial keratotomy. Arch Ophthalmol 1992;110:351-6. 